\title{
iTRAQ-based quantitative proteomic analysis and bioinformatics study of proteins in retinoblastoma
}

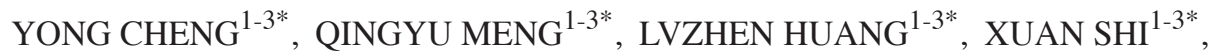 \\ JING HOU ${ }^{1-3}$, XIAOXIN LI ${ }^{1-3}$ and JIANHONG LIANG ${ }^{1-3}$ \\ ${ }^{1}$ Ophthalmology Department, Peking University People's Hospital; ${ }^{2}$ Key Laboratory of Vision Loss and Restoration, \\ Ministry of Education; ${ }^{3}$ Beijing Key Laboratory of Diagnosis and Therapy of Retinal and Choroid Diseases, \\ Peking University People's Hospital, Beijing 100044, P.R. China
}

Received December 23, 2015; Accepted May 16, 2017

DOI: $10.3892 / \mathrm{ol} .2017 .7221$

\begin{abstract}
The aim of the present study was to analyze proteins in the aqueous humor $(\mathrm{AH})$ of patients' retinoblastoma $(\mathrm{RB})$, and investigate their potential role in $\mathrm{RB}$ using the comparative proteomic technique of isobaric tags for relative and absolute quantitation (iTRAQ) coupled with offline two-dimensional liquid chromatography-tandem mass spectrometry. A total of $0.1 \mathrm{ml} \mathrm{AH}$ was collected from 10 children with RB (mean age, 3.8 years; range, 2-5 years) and patients with senile cataracts (mean age, 70.4 years; range, 65-79 years), which was used as the control. iTRAQ was used to analyze proteins in the $\mathrm{AH}$ of patients and controls. Proteins with a fold change of $>1.20$ or $<0.83$ were considered to be significantly differentially expressed (with corrected $\mathrm{P}<0.05$ ). The identified proteins were subjected to subsequent gene ontology (GO) analysis using the DAVID database. A total of 83 proteins that were expressed differently between the controls and patients' $\mathrm{AH}$ samples were identified using iTRAQ analysis. Of these proteins, 44 were upregulated and 39 were downregulated. On the basis of biological processes in GO, the identified proteins were primarily involved in glycoprotein, amyloid acute-inflammatory and defensive responses. Among these proteins, pigment epithelium-derived factor serves a potential role in the treatment of $\mathrm{RB}$, and stimulated by retinoic acid 6 may serve as a potential protein involved in $\mathrm{RB}$ development. To the best of our knowledge, the present study is the first to identify 83 proteins associated with RB using iTRAQ technology. The results of the present study will aid in furthering
\end{abstract}

Correspondence to: Professor Jianhong Liang, Ophthalmology Department, Peking University People's Hospital, 11 Xizhimen South Avenue, Xicheng, Beijing 100044, P.R. China

E-mail: drliangjianhong@126.com

*Contributed equally

Key words: retinoblastoma, isobaric tags for relative and absolute quantitation analysis, gene ontology analysis, pigment epithelium-derived factor, stimulated by retinoic acid 6 the understanding of RB and developing novel therapy targets in the future.

\section{Introduction}

Retinoblastoma (RB) is the most common primary type of intraocular malignancy among children. It originates from the primitive stem cells in the nuclear layer of the retina. Its prevalence is between $1 / 15,000$ and 1/18,000 cases/people, with $95 \%$ of cases occurring before the age of 5 years (1). The main symptoms of RB are leukokoria and strabismus. The RB transcriptional corepressor 1 (RB1) gene located on chromosome 13 is associated with RB. The RB1 gene, which produces the RB protein, serves an important role in regulating and controlling the cell cycle (2). Loss-of-function mutations in RB1 disrupt the cell cycle and have been revealed to be an important initiating event prior to the development of RB (3).

Besides the RB1 gene, other genes and proteins have been observed to serve important roles in the pathogenesis of RB. For example, $1 \%$ of all cases of RB have high levels of MYCN proto-oncogene bHLH transcription factor amplification and no RB mutations. Furthermore, a previous study demonstrated that proteins associated with the redox signaling pathways are involved in RB pathogenesis (4).

In order to further investigate the pathogenesis of RB, the present study performed the quantitative proteomic strategy of using isobaric tags for relative and absolute quantitation (iTRAQ) coupled with two-dimensional liquid chromatography-tandem mass spectrometry (MS) in order to identify associated proteins. The main advantage of the iTRAQ technique for proteomic analysis is that its multiplexing capability allows various protein samples to be simultaneously quantified with a control-standard sample that is processed in the same run (5). The insights the present study have gained may be used for further research of RB in pursuit of a novel therapy target.

\section{Materials and methods}

Subjects. Patients and control subjects were recruited from the Department of Ophthalmology, Peking University People's 
Hospital (Beijing, China). The present study was approved by the Clinic Institutional Review Board of Peking University People's Hospital and complied with the Declaration of Helsinki. Written informed consent was obtained from all patients prior to enrollment in the present study. A total of 10 patients ( 2 women and 8 men; mean age, 3.8 years; range, 2-5 years) diagnosed with RB between September 2014 and March 2015 were included and 10 patients with cataracts (3 women and 7 men; mean age, 70.4 years; range, 65-79 years) were recruited as controls. The inclusion criteria were as follows: Diagnosis of group D RB in accordance with the International Classification of Retinoblastoma (6) with clear optical media in poor responders to chemotherapy, laser or cryotherapy. Patients with RB or control subjects with a history of any systemic or ocular disorder or condition (including ocular surgery, trauma and disease) were excluded from the current study.

Sample collection. Aqueous humor (AH) samples from patients with RB and control subjects were collected. The whole procedure was performed using a microscope viewing through a dilated pupil, followed by intravitreal injections. As previously described (7), anterior chamber paracentesis was performed through a clear cornea limbus track created with a $25 \mathrm{G}$ MVR blade without perforating the Descemet's membrane. A $32 \mathrm{G}$ needle mounted on a tuberculin syringe was then introduced through the track tangentially into the anterior chamber periphery, parallel to the iris. A volume of $0.1 \mathrm{ml}$ aqueous fluid was aspirated, registered and stored at $-80^{\circ} \mathrm{C}$ until processing. For patients with RB, three cycles of freeze and thaw (6 sec each) were applied at the injection site at the time of removal of the needle.

Protein extraction and digestion. Total AH protein concentration was determined using a Bradford protein assay kit (Bio-Rad Laboratories, Inc., Hercules, CA, USA), according to the manufacturer's protocol and as described in a previous study (8). Each sample $(200 \mu \mathrm{g})$ was reconstituted in SDS-PAGE sample buffer with 5\% $\beta$-mercaptoethanol, and electrophoresed on a (10-14.5\%) SDS-PAGE precast gel (Criterion; Bio-Rad Laboratories, Inc.). A total of nine gel slices were excised from each lane. In-gel digestion was performed as previously reported (8). Briefly, the excised bands were de-stained using $40 \mathrm{mM}$ ammonium bicarbonate in $50 \%$ acetonitrile $(\mathrm{ACN})$ solution $\left(45^{\circ} \mathrm{C}\right.$ for $\left.20 \mathrm{~min}\right)$. The de-stained gel bands were then subjected to reduction using $5 \mathrm{mM}$ dithiothreitol $\left(60^{\circ} \mathrm{C}\right.$ for $\left.45 \mathrm{~min}\right)$, followed by alkylation using $10 \mathrm{mM}$ iodoacetamide $\left(56^{\circ} \mathrm{C}\right.$ for $\left.60 \mathrm{~min}\right)$. The gel pieces were dehydrated using $100 \% \mathrm{ACN}$, followed by digestion with trypsin (modified sequencing grade; Promega Corporation, Madison, WI, USA) at $37^{\circ} \mathrm{C}$ for $12-16 \mathrm{~h}$. The peptides were extracted from the gel fragments by treating the gel bands twice with $0.4 \%$ formic acid and $3 \% \mathrm{ACN}$ solution once with $0.4 \%$ formic acid and 50\% ACN solution and finally with $100 \%$ ACN solution (all at room temperature for $15 \mathrm{~min}$ each). The samples were labeled with iTRAQ ${ }^{\circledR}$ reagents by adding the contents of the iTRAQ Reagent-8Plex Multiplex kit (Applied Biosystems; Thermo Fisher Scientific, Inc., Waltham, MA, USA) to the sample solutions. The two samples were iTRAQ-labeled as follows: R0 (RB) and C4 (Control).
Two-dimensional liquid chromatography-electrospray ionization MS. Sample analysis was performed using a QTRAP 5500 system (Applied Biosystems; Thermo Fisher Scientific, Inc.) to generate MS and tandem MS (MS/MS) data. Peptides were loaded onto a Kinetex 100.0x2.1-mm C18 column (300 ̊, $2.6 \mu \mathrm{m}$; Phenomenex, Torrance, CA, USA) and then submitted to mobile-phase elution in buffer A $(0.1 \%$ formic acid in water) and buffer B (0.1\% formic acid in acetonitrile), according to the manufacturer's protocol. The peptides were eluted at a flow rate of $400 \mu \mathrm{l} / \mathrm{min}$. The liquid chromatography eluent was directed to an electrospray ionization source for quantitative time-of-flight MS analysis. Electrospray ionization was performed for information-dependent acquisition in positive-ion mode with a spray voltage of $1.8 \mathrm{kV}$ and a selected mass range of 350-2,000 m/z. The QTRAP 5500 system was operated in data-dependent acquisition mode. The three most abundantly charged peptides above a 5-count threshold were selected for MS/MS.

Database search. All MS/MS samples were analyzed using Mascot (version 2.4.1; Matrix Science, London, UK). Mascot was set up to search the Uniprot2014_human database (www. uniprot.org; accessed January 2014). The analysis and search parameters were as follows: Trypsin as the digestion enzyme with allowance for a maximum of one missed cleavage; Carbamidomethyl (C) as a fixed modification; Oxidation (M), Gln $\rightarrow$ Pyro-Glu (N-term Q) and iTRAQplex modification (K, $\mathrm{Y}$ and $\mathrm{N}$ termini) as a variable modification; peptide mass tolerance of $15 \mathrm{ppm}$; and fragment mass tolerance of $20 \mathrm{mmu}$.

Expression changes of the identified peptides in human AH were determined and compared with the controls using the iTRAQ reporter ion intensities. Based on the relative quantification and statistical analysis, a 1.2-fold change cut-off was selected to categorize proteins as significantly altered. Therefore, proteins with iTRAQ ratios $>1.2$ were considered to be upregulated, whereas those with iTRAQ ratios $<0.83$ were considered to be downregulated.

Bioinformatics analysis. In order to characterize the function of the proteins identified in the quantitative proteomics analysis, information from the DAVID Bioinformatics website (https://david.ncifcrf.gov/) was applied to the functional enrichment and gene ontology $(\mathrm{G})$ analyses, as previously described (9). In the present study, the GO categories with $\mathrm{P}<0.05$ were considered to indicate a statistically significant expression in patients with RB.

\section{Results}

Protein identification in AH. Proteins with corrected $\mathrm{P}<0.05$ and a fold change of $>1.2$ or $<0.83$ were considered to be significantly differentially expressed. In total, 83 proteins were identified in the AH of patients with RB by iTRAQ analysis (Table I). Of these proteins, 44 were upregulated and 39 were downregulated. The proteins with an increased fold change of $>2.0$ included the following: Vitamin D-binding protein, angiotensinogen, carbonic anhydrase 1 , Ig $\kappa$-chain V-III region $\mathrm{B} 6$, $\operatorname{Ig} \alpha-1$ chain $\mathrm{C}$ region, $\alpha$-1-antitrypsin, prothrombin, anti-thrombin-III stimulated by retinoic acid 6 (STRA6), fibrinogen $\lambda$-chain, Ig $\lambda-2$ chain $C$ region, thyroxine-binding 
Table I. Proteins identified in the aqueous humor of patients with retinoblastoma using iTRAQ analysis.

\begin{tabular}{|c|c|c|}
\hline Fold change & Protein & Description \\
\hline 0.269 & CRYGS & $\beta$-crystallin $S$ \\
\hline 0.351 & PSAP & Prosaposin \\
\hline 0.387 & GPX3 & Glutathione peroxidase 3 \\
\hline 0.389 & CTSD & Cathepsin D \\
\hline 0.478 & RBP3 & Retinol-binding protein 3 \\
\hline 0.52 & LGALS3BP & Galectin-3-binding protein \\
\hline 0.522 & LDHA & L-lactate dehydrogenase $\alpha$-chain \\
\hline 0.528 & CLSTN1 & Calsyntenin-1 \\
\hline 0.571 & $\mathrm{C} 4 \mathrm{~A}$ & Complement C4-A \\
\hline 0.572 & Ig $\kappa$ chain V-III region SIE & Ig $\kappa$-chain V-III region SIE \\
\hline 0.593 & Ig $\kappa$ chain V-I region Roy & Ig $\kappa$-chain V-I region Roy \\
\hline 0.594 & LACRT & Extracellular glycoprotein lacritin \\
\hline 0.596 & FBLN & Fibulin-1 \\
\hline 0.598 & LUM & Lumican \\
\hline 0.614 & SERPING1 & Plasma protease $\mathrm{C} 1$ inhibitor \\
\hline 0.621 & APLP & Amyloid-like protein 2 \\
\hline 0.625 & PTGDS & Prostaglandin-H2 D-isomerase \\
\hline 0.631 & CLEC3B & Tetranectin \\
\hline 0.66 & $\mathrm{~A} 2 \mathrm{M}$ & $\alpha$-2-macroglobulin \\
\hline 0.681 & KRT9 & Keratin, type I cytoskeletal 9 \\
\hline 0.682 & SPON1 & Spondin-1 \\
\hline 0.687 & ENPP & Ectonucleotide pyrophosphatase/phosphodiesterase family member 2 \\
\hline 0.727 & GSN & Gelsolin \\
\hline 0.73 & LYZ & Lysozyme C \\
\hline 0.739 & KRT14 & Keratin, type I cytoskeletal 14 \\
\hline 0.743 & $\mathrm{CP}$ & Ceruloplasmin \\
\hline 0.75 & $\mathrm{RBP}$ & Retinol-binding protein 4 \\
\hline 0.758 & Ig $\lambda$ chain $\mathrm{V}$-I region $\mathrm{NEW}$ & Ig $\lambda$-chain V-I region NEW \\
\hline 0.773 & SERPINF1 & Pigment epithelium-derived factor \\
\hline 0.777 & IGFBP7 & Insulin-like growth factor-binding protein 7 \\
\hline 0.787 & KRT10 & Keratin, type I cytoskeletal 10 \\
\hline 0.788 & TTR & Transthyretin \\
\hline 0.803 & CST3 & Cystatin-C \\
\hline 0.814 & $\operatorname{Ig} \lambda$ chain $\mathrm{V}$-III region $\mathrm{SH}$ & Ig $\lambda$-chain $\mathrm{V}$-III region $\mathrm{SH}$ \\
\hline 0.82 & KRT1 & Keratin, type II cytoskeletal 1 \\
\hline 0.82 & $\mathrm{C} 2$ & Complement $\mathrm{C} 2$ \\
\hline 0.82 & IGLL5 & Immunoglobulin $\lambda$-like polypeptide 5 \\
\hline 0.832 & HP & Haptoglobin \\
\hline 0.833 & KRT2 & Keratin, type II cytoskeletal 2 epidermal \\
\hline 1.238 & Ig heavy chain V-III region GAL & Ig heavy chain V-III region GAL \\
\hline 1.24 & AZGP1 & Zinc- $\alpha$-2-glycoprotein \\
\hline 1.261 & ORM1 & $\alpha$-1-acid glycoprotein 1 \\
\hline 1.261 & Ig heavy chain V-I region EU & Ig heavy chain V-I region EU \\
\hline 1.274 & OPTC & Opticin OS=Homo sapiens \\
\hline 1.278 & AMBP & Protein AMBP \\
\hline 1.288 & APOA1 & Apolipoprotein A-I \\
\hline 1.293 & LRG1 & Leucine-rich $\alpha$-2-glycoprotein \\
\hline 1.306 & Ig heavy chain V-III region BRO & Ig heavy chain V-III region BRO \\
\hline 1.321 & CHI3L1 & Chitinase-3-like protein 1 \\
\hline 1.354 & VTN & Vitronectin \\
\hline 1.378 & HPX & Hemopexin \\
\hline 1.378 & IGHG1 & Ig $\gamma-1$ chain $\mathrm{C}$ region \\
\hline
\end{tabular}


Table I. Continued.

\begin{tabular}{|c|c|c|}
\hline Fold change & Protein & Description \\
\hline 1.388 & LCN1P1 & Putative lipocalin 1-like protein 1 \\
\hline 1.408 & APOA4 & Apolipoprotein A-IV \\
\hline 1.457 & ALB & Serum albumin \\
\hline 1.468 & ORM2 & $\alpha$-1-acid glycoprotein 2 \\
\hline 1.482 & A1BG & $\alpha$-1B-glycoprotein \\
\hline 1.538 & HBD & Hemoglobin subunit delta \\
\hline 1.546 & FGA & Fibrinogen $\alpha$ chain \\
\hline 1.547 & Ig heavy chain V-III region TIL & Ig heavy chain V-III region TIL \\
\hline 1.549 & HRG & Histidine-rich glycoprotein \\
\hline 1.55 & IGHM & Ig $\mu$-chain $\mathrm{C}$ region \\
\hline 1.552 & SMTN & Smoothelin \\
\hline 1.642 & EFEMP & EGF-containing fibulin-like extracellular matrix protein 1 \\
\hline 1.705 & $\mathrm{HBB}$ & Hemoglobin subunit $\beta$ \\
\hline 1.746 & Ig heavy chain V-I region $\mathrm{HG} 3$ & Ig heavy chain V-I region HG3 \\
\hline 1.765 & NOL6 & Nucleolar protein 6 \\
\hline 1.768 & KNG1 & Kininogen-1 \\
\hline 1.975 & SERPINA3 & $\alpha$-1-antichymotrypsin \\
\hline 2.012 & $\mathrm{GC}$ & Vitamin D-binding protein \\
\hline 2.089 & AGT & Angiotensinogen \\
\hline 2.124 & CA1 & Carbonic anhydrase 1 \\
\hline 2.191 & Ig $\kappa$ chain $\mathrm{V}$-III region $\mathrm{B} 6$ & Ig $\kappa$-chain V-III region B6 \\
\hline 2.218 & IGHA1 & Ig $\alpha-1$ chain $\mathrm{C}$ region \\
\hline 2.267 & SERPINA1 & $\alpha-1$-antitrypsin \\
\hline 2.429 & $\mathrm{~F} 2$ & Prothrombin \\
\hline 2.437 & SERPINC1 & Antithrombin-III \\
\hline 2.479 & STRA6 & Stimulated by retinoic acid gene 6 protein homolog \\
\hline 2.573 & FGG & Fibrinogen $\gamma$ chain \\
\hline 2.605 & IGHG2 & $\operatorname{Ig} \gamma-2$ chain $\mathrm{C}$ region \\
\hline 2.973 & SERPINA7 & Thyroxine-binding globulin \\
\hline 2.98 & AHSG & $\alpha$-2-HS-glycoprotein \\
\hline 3.146 & HBA1 & Hemoglobin subunit $\alpha$ \\
\hline
\end{tabular}

iTRAQ, isobaric tags for relative and absolute quantitation analysis.

globulin, $\alpha$-2-HS-glycoprotein and hemoglobin subunit $\alpha$. The five proteins with a decreased fold change of $>0.5$ were $\beta$-crystallin $\mathrm{S}$, prosaposin, glutathione peroxidase 3, cathepsin D and retinol-binding protein (RBP) 3 .

GO analysis. In order to identify the functions of proteins identified using the iTRAQ technique, the present study performed GO analysis with the assistance of DAVID Bioinformatics Resources. The DAVID classification of proteins by biological process demonstrated that the proteins were primarily involved in the defensive (27.3\%), inflammatory (27.3\%) and acute-phase $(27.3 \%)$ responses, and the response to wounding (16.7\%) (Fig. 1). On the basis of molecular function annotations, the proteins in the present study were implicated in endopeptidase inhibitor activity (21.2\%), peptidase inhibitor (21.2\%), enzyme inhibitor (21.2\%), serine-type endopeptidase inhibitor $(15.2 \%)$, structural molecule $(15.2 \%)$, lipid binding (12.1\%) and carbohydrate binding (10.6\%) activities (Fig. 2). In the cellular component ontology, the present study revealed that the majority of enriched categories were associated with extracellular construction, including extracellular region $(75.8 \%)$, extracellular region part (56.1\%) and extracellular space (50\%) (Fig. 3). Functional annotation clustering demonstrated that they belong to glycoprotein, amyloid, acute-inflammatory and defense responses (Fig. 4).

\section{Discussion}

RB has become curable in the majority of cases in children, so long as there is early diagnosis and accurate prognosis. In order to investigate RB, the present study performed iTARQ analysis to identify proteins that may serve a role in RB in children. A total of 44 upregulated proteins and 39 downregulated proteins were identified. Functional annotation clustering revealed that they belong to glycoprotein, amyloid, acute-inflammatory and defense responses. Among these proteins, the present study 


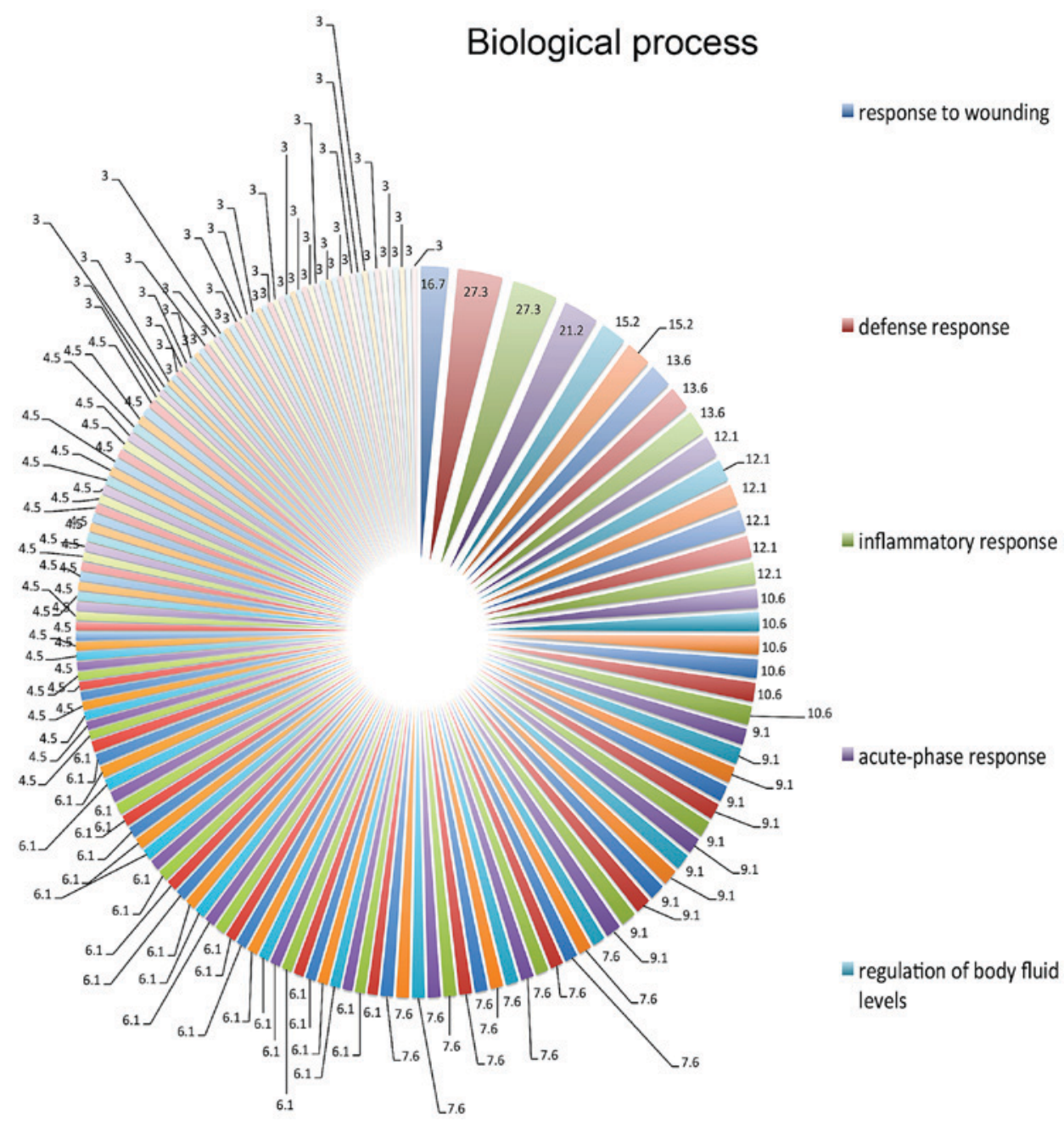

Figure 1. Functional classification of proteomic data by bioinformatics analysis. The biological process categories. Categorizations are based on information provided by the online resource, Gene Ontology, and DAVID Bio-informatics Resources.

\section{Molecular function}

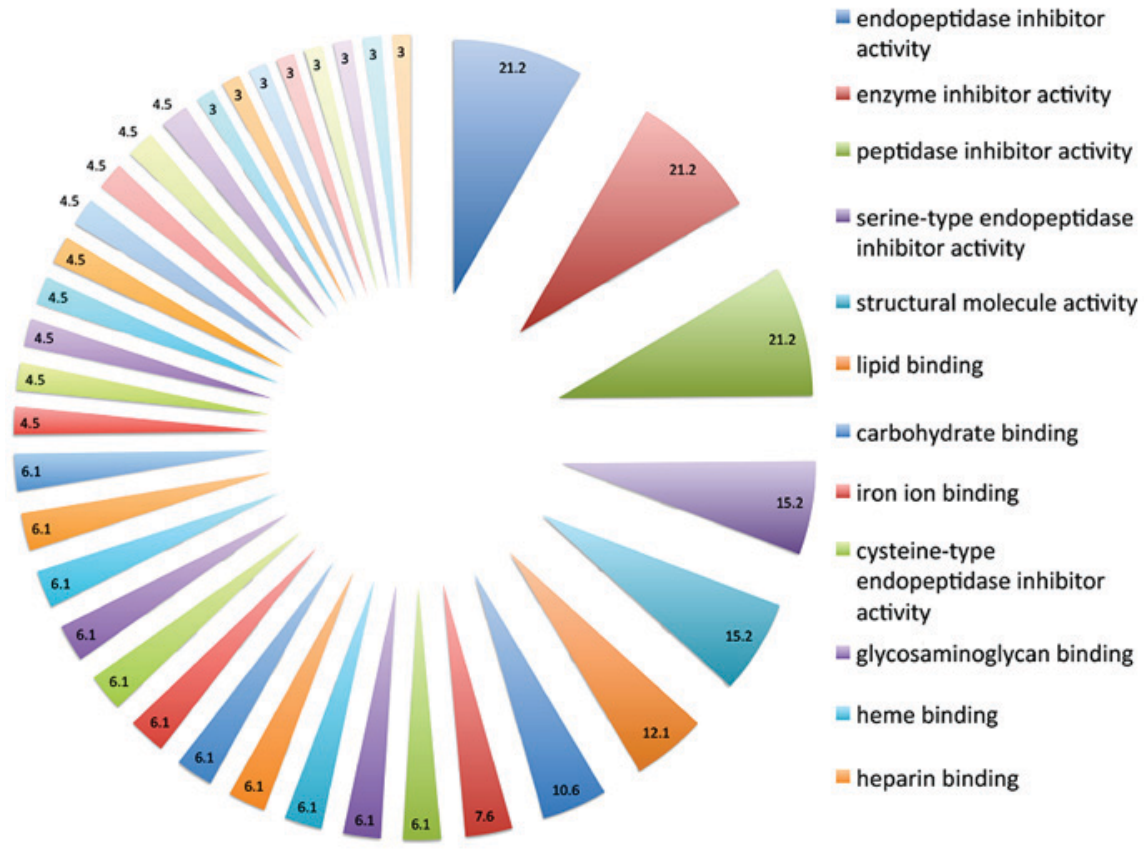

Figure 2. Functional classification of proteomic data by bioinformatics analysis. The molecular function categories. Categorizations are based on information provided by the online resource, Gene Ontology, and DAVID Bio-informatics Resources. 


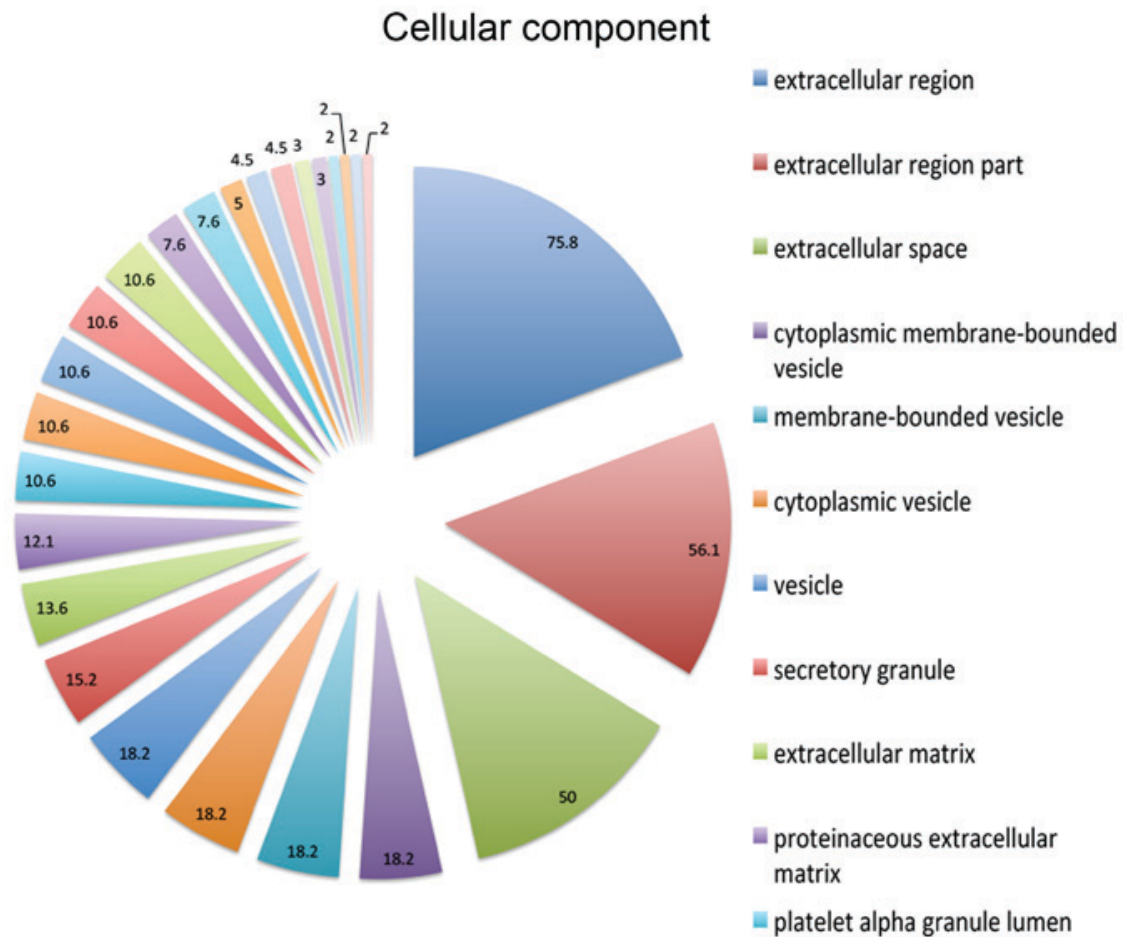

Figure 3. Functional classification of proteomic data by bioinformatics analysis. The cellular component categories. Categorizations are based on information provided by the online resource, Gene Ontology, and DAVID Bio-informatics Resources.

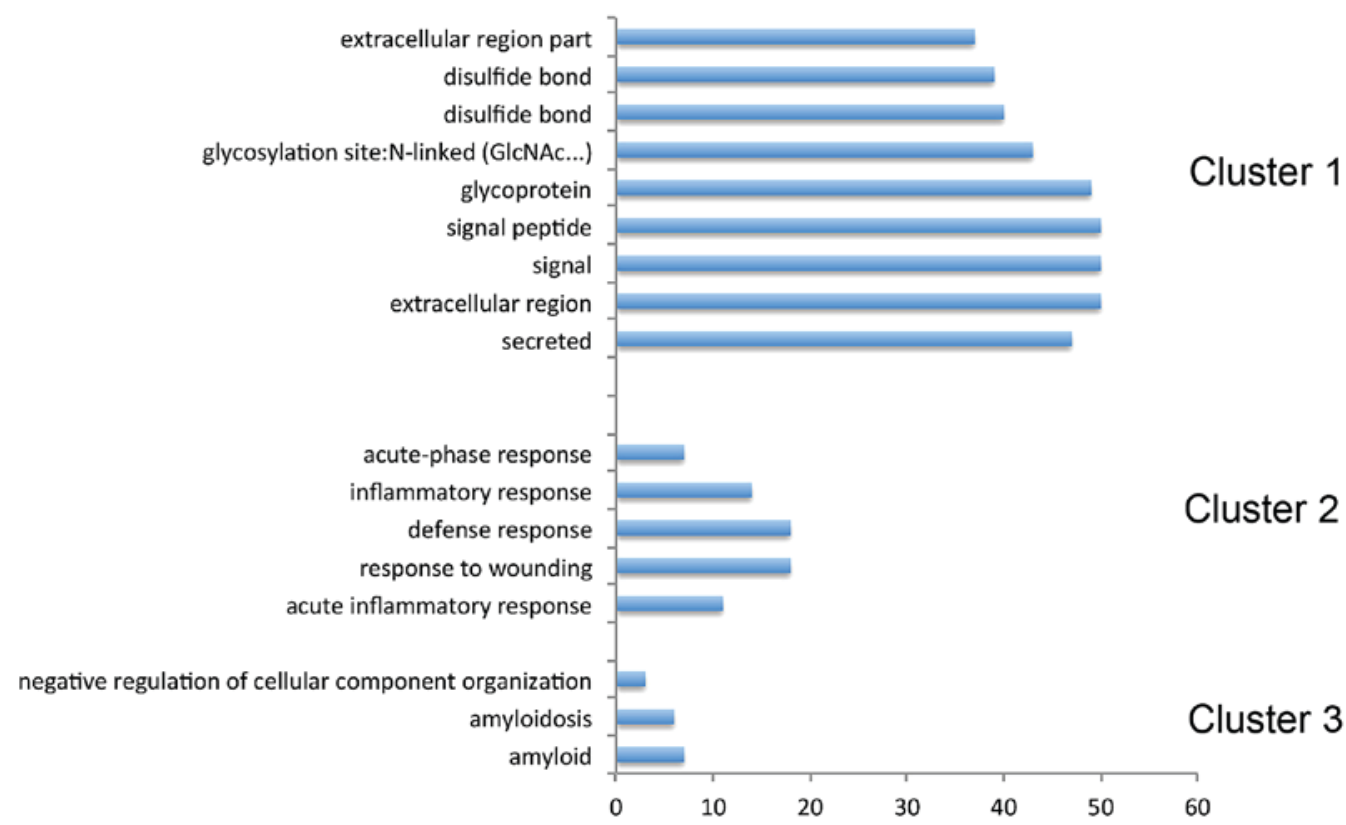

Figure 4. Functional annotation clustering determined using DAVID Bioinformatics Resources with respect to the target proteins. The x-axis represents the number of proteins and the $y$-axis represents the ontology categories.

speculated that pigment epithelium-derived factor precursor (PEDF) serves a potential role in the treatment of RB and STRA6 may be involved in RB development.

PEDF is a member of the serine protease inhibitor (serpin) superfamily. As a nerotrophic factor, PEDF promotes the differentiation of RB cells and other tumor cells of neuronal origin (10). It has potent anti-angiogenesis activity, and it has been revealed that PEDF may delay tumor growth and decrease the expression level of vascular endothelial growth factor (VEGF) (11). Of note, PEDF has been demonstrated to be an inhibitor of tumor cell invasion, migration and metastasis in vitro (12), and in numerous in vivo models (13-15). It is also known that the Fas cell surface death receptor (Fas)/Fas-L/caspase-8 apoptotic signaling pathway is involved in the apoptosis of PEDF-induced endothelial cells (16). Dawson et al (11) identified PEDF as a potent inhibitor of angiogenesis in the eye. A number of studies have identified that PEDF overexpression could prevent ocular 
neovascularization, and delay photoreceptor and neural retinal cell death in vivo (17-19). Yang et al (20) revealed that PEDF inhibited tumor angiogenesis, as microvessel density was demonstrated to have decreased in PEDF-treated tumor tissues. Further research demonstrated that PEDF may downregulate VEGF expression in vitro and in vivo by inhibition of hypoxia-inducible factor- $1 \alpha$. The anti-angiogenic effect of PEDF makes it an excellent candidate as a therapy target for $\mathrm{RB}$. The present study revealed that the expression level of PEDF was lower in RB compared with control samples, which was consistent with the results in a previous study (20). These results suggest the potentially pivotal role that PEDF may serve in RB treatment.

STRA6 is widely expressed during embryonic development and in adult organ systems (21). STRA6 is the receptor of RBP and transports retinol from extracellular RBP into cells (22). It also serves as a cytokine and transduces a signaling cascade via Janus kinase 2, and the transcription factors, signal transducer and activator of transcription (STAT) 3 and STAT5, leading to induction of STAT target gene expression that promote oncogenic transformation (23). Overexpression of STRA6 has been observed in numerous types of human cancer, including Wilm kidney tumors, melanomas, and colorectal, ovarian and endometrium cancer (24). In vitro, STRA6 may facilitate cell proliferation, migration and invasion in cells. However, it was recently reported that STRA6 contributes to p53-induced apoptosis in response to DNA damage (25). The present study demonstrated that STRA6 was upregulated in patients with $\mathrm{RB}$, and further research is required in order to identify the function of STRA6 in the development of RB.

The GO analysis of the present study revealed that the identified proteins were involved in glycoprotein, amyloid, acute-inflammatory and defense responses. Glycosylation serves an important role in posttranslational modifications, and modulates the physical, chemical and biological properties of a protein $(26,27)$. Glycan structures are important for numerous processes, including protein-protein interactions, protein trafficking and folding, immune recognition, cell adhesion, and migration and inter-cellular signaling (28). A number of studies have identified that aberrant glycans may serves an essential role in cancer biology by mediating tumor cell adhesion, motility and invasiveness (29-31). The first group identified in the functional clustering analysis involved glycoproteins. $\alpha-1 \mathrm{~B}$-glycoprotein (A1BG) is a plasma glycoprotein that has sequence similarity to the variable regions of certain immunoglobulin supergene family member proteins. Recent proteomics studies have revealed that A1BG may act as a biomarker for numerous types of tumors, including bladder (32) and pancreatic (33) cancer. Leucine-rich $\alpha$-2-glycoprotein (LRG1) is a secreted glycoprotein of the leucine-rich repeat family. It was reported that LRG1 is associated with cancer metastasis and poor prognosis, resulting from its effects on promoting cell invasion, angiogenesis, and migration (33). Chitinase 3-like 1 (CHI3L1) is a member of the glycosyl hydrolase 18 family. CHI3L1 is considered to serve a role in the process of inflammation and tissue remodeling (34). A previous study demonstrated the association of high serum CHI3L1 expression level with poor patient prognosis and short survival time in human solid tumors, including lung cancer and liver cancer (35).
Additionally, the present study indicated that proteins involved in acute phase responses are associated with RB. The acute phase response is a rapid inflammatory response that provides protection against microorganisms using non-specific defense mechanisms $(36,37)$. It was observed that inflammation may serve a dominant role in the pathogenesis of various types of cancer $(38,39)$. Fibrinogen $\gamma$, serpin family A member 1 (SerpinA1) and orosomucoid 2 (ORM2) are all acute-phase associated proteins. Fibrinogen is involved in numerous processes, including blood clotting, fibrinolysis, the inflammatory response and wound healing. Previously, the role of fibrinogen and fibrinogen degradation products in carcinogenesis of certain tumor types has been suggested $(40,41)$. Proteomic analysis has also demonstrated that fibrinogen $\gamma$ is overexpressed in patients with pancreatic cancer (42). SerpinA1 is produced in the liver and secreted into serum. It has been reported to exhibit an invasive and metastatic capacity in lung cancer, gastric cancer, and colorectal cancer $(43,44)$. Previous studies have revealed that SerpinA1 may serve as a biomarker for gastric cancer as it induces the invasion and migration of gastric cancer cells, and its expression is associated with the progression of gastric cancer (45). ORM2 is an important acute phase plasma protein due to its increased abundance during acute inflammation. ORM2 may function in the modulation of the immune system in the acute phase. Upregulation of ORM2 has been reported in patients with colorectal cancer (46).

In conclusion, to the best of our knowledge, the present study is the first to identify proteins associated with RB using iTRAQ technology. The results of the present study may aid in providing an improved understanding of RB and contribute to developing a novel therapy target in the future. Further studies are required to explore the function of proteins in $\mathrm{RB}$ development.

\section{Acknowledgements}

The present study was supported by Peking University People's Hospital Research and Development Funds (grant no. RDC-2014-24).

\section{References}

1. Bishop JO and Madson EC: Retinoblastoma. Review of the current status. Surv Ophthalmol 19: 342-366, 1975.

2. Knudson AG: Cancer genetics. Am J Med Genet 111: 96-102, 2002.

3. Corson TW and Gallie BL: One hit, two hits, three hits, more? Genomic changes in the development of retinoblastoma. Genes Chromosomes Cancer 46: 617-634, 2007.

4. Vandhana S, Lakshmi TS, Indra D, Deepa PR and Krishnakumar S: Microarray analysis and biochemical correlations of oxidative stress responsive genes in retinoblastoma. Curr Eye Res 37: 830-841, 2012.

5. Tannu NS and Hemby SE: Methods for proteomics in neuroscience. Prog Brain Res 158: 41-82, 2006.

6. Shields CL, Mashayekhi A, Au AK, Czyz C, Leahey A Meadows AT and Shields JA: The International Classification of Retinoblastoma predicts chemoreduction success. Ophthalmology 113: 2276-2280, 2006.

7. Munier FL, Soliman S, Moulin AP, Gaillard MC, Balmer A and Beck-Popovic M: Profiling safety of intravitreal injections for retinoblastoma using an anti-reflux procedure and sterilisation of the needle track. Br J Ophthalmol 96: 1084-1087, 2012.

8. Duan X, Lu Q, Xue P, Zhang H, Dong Z, Yang F and Wang N: Proteomic analysis of aqueous humor from patients with myopia. Mol Vis 14: 370-377, 2008. 
9. Huang da W, Sherman BT and Lempicki RA: Systematic and integrative analysis of large gene lists using DAVID bioinformatics resources. Nat Protoc 4: 44-57, 2009.

10. Steele FR, Chader GJ, Johnson LV and Tombran-Tink J: Pigment epithelium-derived factor: Neurotrophic activity and identification as a member of the serine protease inhibitor gene family. Proc Natl Acad Sci USA 90: 1526-1530, 1993.

11. Dawson DW, Volpert OV, Gillis P, Crawford SE, Xu H, Benedict W and Bouck NP: Pigment epithelium-derived factor: A potent inhibitor of angiogenesis. Science 285: 245-248, 1999.

12. Subramanian P, Deshpande M, Locatelli-Hoops S, MoghaddamTaaheri S, Gutierrez D, Fitzgerald DP, Guerrier S, Rapp M, Notario V and Becerra SP: Identification of pigment epitheliumderived factor protein forms with distinct activities on tumor cell lines. J Biomed Biotechnol 2012: 425907, 2012.

13. Guan M, Jiang H, Xu C, Xu R, Chen Z and Lu Y: Adenovirusmediated PEDF expression inhibits prostate cancer cell growth and results in augmented expression of PAI-2. Cancer Biol Ther 6: 419-425, 2007.

14. Guan M, Pang CP, Yam HF, Cheung KF, Liu WW and Lu Y: Inhibition of glioma invasion by overexpression of pigment epithelium-derived factor. Cancer Gene Ther 11: 325-332, 2004.

15. Orgaz JL, Ladhani O, Hoek KS, Fernández-Barral A, Mihic D, Aguilera O, Seftor EA, Bernad A, Rodríguez-Peralto JL, Hendrix MJ, et al: 'Loss of pigment epithelium-derived factor enables migration, invasion and metastatic spread of human melanoma'. Oncogene 28: 4147-4161, 2009.

16. Li L, Yao YC, Fang SH, Ma CQ, Cen Y, Xu ZM, Dai ZY, Li C, Li S, Zhang T, et al: Pigment epithelial-derived factor (PEDF)triggered lung cancer cell apoptosis relies on $\mathrm{p} 53$ protein-driven Fas ligand (Fas-L) up-regulation and Fas protein cell surface translocation. J Biol Chem 289: 30785-30799, 2014.

17. Bouck N: PEDF: Anti-angiogenic guardian of ocular function. Trends Mol Med 8: 330-334, 2002

18. Mori K, Duh E, Gehlbach P, Ando A, Takahashi K, Pearlman J, Mori K, Yang HS, Zack DJ, Ettyreddy D, et al: Pigment epithelium-derived factor inhibits retinal and choroidal neovascularization. J Cell Physiol 188: 253-263, 2001.

19. Amaral J and Becerra SP: Effects of human recombinant PEDF protein and PEDF-derived peptide 34-mer on choroidal neovascularization. Invest Ophthalmol Vis Sci 51: 1318-1326, 2010

20. Yang H, Cheng R, Liu G, Zhong Q, Li C, Cai W, Yang Z, Ma J, Yang $\mathrm{X}$ and Gao G: PEDF inhibits growth of retinoblastoma by anti-angiogenic activity. Cancer Sci 100: 2419-2425, 2009.

21. Chazaud C, Bouillet P, Oulad-Abdelghani M and Dollé P: Restricted expression of a novel retinoic acid responsive gene during limb bud dorsoventral patterning and endochondral ossification. Dev Genet 19: 66-73, 1996.

22. Kawaguchi R, Yu J, Honda J, Hu J, Whitelegge J, Ping P, Wiita P, Bok D and Sun H: A membrane receptor for retinol binding protein mediates cellular uptake of vitamin A. Science 315: $820-825,2007$

23. Berry DC, Levi L and Noy N: Holo-retinol-binding protein and its receptor STRA6 drive oncogenic transformation. Cancer Res 74: 6341-6351, 2014.

24. Szeto W, Jiang W, Tice DA, Rubinfeld B, Hollingshead PG, Fong SE, Dugger DL, Pham T, Yansura DG, Wong TA, et al: Overexpression of the retinoic acid-responsive gene Stra6 in human cancers and its synergistic induction by Wnt-1 and retinoic acid. Cancer Res 61: 4197-4205, 2001.

25. Carrera S, Cuadrado-Castano S, Samuel J, Jones GD, Villar E, Lee SW and Macip S: Stra6, a retinoic acid-responsive gene, participates in p53-induced apoptosis after DNA damage. Cell Death Differ 20: 910-919, 2013.

26. Dube DH and Bertozzi CR: Glycans in cancer and inflammation-potential for therapeutics and diagnostics. Nat Rev Drug Discov 4: 477-488, 2005

27. Bertozzi CR and Kiessling LL: Chemical glycobiology. Science 291: 2357-2364, 2001
28. Chen S, LaRoche T, Hamelinck D, Bergsma D, Brenner D, Simeone D, Brand RE and Haab BB: Multiplexed analysis of glycan variation on native proteins captured by antibody microarrays. Nat Methods 4: 437-444, 2007.

29. Taniguchi N and Kizuka Y: Glycans and cancer: Role of $\mathrm{N}$-glycans in cancer biomarker, progression and metastasis, and therapeutics. Adv Cancer Res 126: 11-51, 2015.

30. Pinho SS and Reis CA: Glycosylation in cancer: Mechanisms and clinical implications. Nat Rev Cancer 15: 540-555, 2015.

31. Compagno D, Gentilini LD, Jaworski FM, Pérez IG, Contrufo G and Laderach DJ: Glycans and galectins in prostate cancer biology, angiogenesis and metastasis. Glycobiology 24: 899-906, 2014.

32. Kreunin P, Zhao J, Rosser C, Urquidi V, Lubman DM and Goodison S: Bladder cancer associated glycoprotein signatures revealed by urinary proteomic profiling. J Proteome Res 6: 2631-2639, 2007.

33. Tian M, Cui YZ, Song GH, Zong MJ, Zhou XY, Chen Y and Han JX: Proteomic analysis identifies MMP-9, DJ-1 and A1BG as overexpressed proteins in pancreatic juice from pancreatic ductal adenocarcinoma patients. BMC cancer 8: 241, 2008.

34. Libreros S and Iragavarapu-Charyulu V: YKL-40/CHI3L1 drives inflammation on the road of tumor progression. J Leukoc Biol 98: 931-936, 2015.

35. Wang XW, Cai CL, Xu JM, Jin H and Xu ZY: Increased expression of chitinase 3-like 1 is a prognosis marker for non-small cell lung cancer correlated with tumor angiogenesis. Tumour Biol 36: 901-907, 2015.

36. Kushner I: Regulation of the acute phase response by cytokines. Perspect Biol Med 36: 611-622, 1993.

37. Davalieva K, Kiprijanovska S, Komina S, Petrusevska G, Zografska NC and Polenakovic M: Proteomics analysis of urine reveals acute phase response proteins as candidate diagnostic biomarkers for prostate cancer. Proteome Sci 13: 2, 2015.

38. Hoenerhoff MJ: Inflammation and cancer: Partners in crime Vet J 206: 1-2, 2015.

39. Raposo TP, Beirão BC, Pang LY, Queiroga FL and Argyle DJ: Inflammation and cancer: Till death tears them apart. Vet J 205: 161-174, 2015.

40. Gerner C, Steinkellner W, Holzmann K, Gsur A, Grimm R, Ensinger C, Obrist P and Sauermann G: Elevated plasma levels of crosslinked fibrinogen gamma-chain dimer indicate cancer-related fibrin deposition and fibrinolysis. Thromb Haemost 85: 494-501, 2001.

41. Palumbo JS, Talmage KE, Massari JV, La Jeunesse CM, Flick MJ, Kombrinck KW, Jirousková M and Degen JL: Platelets and fibrin(ogen) increase metastatic potential by impeding natural killer cell-mediated elimination of tumor cells. Blood 105: 178-185, 2005

42. Bloomston M, Zhou JX, Rosemurgy AS, Frankel W, Muro-Cacho CA and Yeatman TJ: Fibrinogen gamma overexpression in pancreatic cancer identified by large-scale proteomic analysis of serum samples. Cancer Res 66: 2592-2599, 2006

43. Yang J, Xiong X, Wang X, Guo B, He K and Huang C: Identification of peptide regions of SERPINA1 and ENOSF1 and their protein expression as potential serum biomarkers for gastric cancer. Tumour Biol 36: 5109-5118, 2015.

44. Kwon CH, Park HJ, Choi JH, Lee JR, Kim HK, Jo HJ, Kim HS, Oh N, Song GA and Park DY: Snail and serpinA1 promote tumor progression and predict prognosis in colorectal cancer. Oncotarget 6: 20312-20326, 2015.

45. Kwon CH, Park HJ, Lee JR, Kim HK, Jeon TY, Jo HJ, Kim DH, Kim GH and Park DY: Serpin peptidase inhibitor clade A member 1 is a biomarker of poor prognosis in gastric cancer. $\mathrm{Br}$ J Cancer 111: 1993-2002, 2014

46. Zhang X, Xiao Z, Liu X, Du L, Wang L, Wang S, Zheng N, Zheng G, Li W, Zhang X, et al: The potential role of ORM2 in the development of colorectal cancer. PLoS One 7: e31868, 2012. 